\title{
Feasibility and Potential Benefits of a Self-Monitoring Enhanced Lifestyle Intervention to Prevent Excessive Gestational Weight Gain in Women Who Are Overweight or Obese
}

Carol Shieh, Ziyi Yang, David M. Haas, Janet S. Carpenter

\begin{abstract}
Objective: To evaluate feasibility and potential benefits of a self-monitoring enhanced lifestyle intervention for excessive gestational weight gain prevention in overweight and obese women. Design: A one-group, prospective design involving eight weeks of healthy eating and physical activity and self-monitoring of weight, nutrition, and walking.

Setting: Recruitment and enrollment in prenatal clinics and self-monitoring at home.

Participants: 22 women of 14-24 gestational weeks, BMI of 25-40, and without medical and psychiatric diseases that affected cognition or walking.
\end{abstract}

Methods: Participants self-monitored weight and nutrition intake for the first 4 weeks and weight, nutrition intake, and walking in the second 4 weeks. Feasibility data were collected weekly (attrition, self-monitoring adherence, program safety, participant feedback) or at the end of the $8^{\text {th }}$ week (satisfaction ratings). Potential benefits included weight, nutrition, and physical activity; measured at baseline (T1), the end of the $4^{\text {th }}$ week (T2), or the end of the $8^{\text {th }}$ week (T3). Results: Attrition rates were $27.3 \%$ by T2 and $40.9 \%$ by T3. Adherence to log return was $100 \%$. No side effects were noted, but food craving was persistent and stress levels were high. Program satisfaction was high. Trends for improved activity and reduced trans fat consumption were seen. Conclusions: Findings indicate the intervention is worthy of further development and testing with a randomized controlled trial.

\section{Keywords:}

Overweight, obesity, pregnancy, gestational weight gain, intervention study, pregnancy weight gain, obesity prevention, self-monitoring, self-weighing, lifestyle management 


\section{Précis statement:}

A self-monitoring enhanced lifestyle intervention to prevent excessive gestational weight gain in overweight and obese women demonstrated feasibility and potential benefits. 


\section{Callouts}

Callout \#1:

About $38 \%$ to $55 \%$ of pregnant women gain more pregnancy weight than what is recommended by the Institute of Medicine.

\section{Callout \#2:}

A self-monitoring enhanced lifestyle intervention may help pregnant women reduce trans fat consumption, increase physical activity, and gain the proper amount of weight.

\section{Callout \#3:}

The intervention showed feasibility in overweight and obese pregnant women and is worthy of further testing in a randomized controlled trial. 
Feasibility and Potential Benefits of a Self-Monitoring Enhanced Lifestyle Intervention to Prevent Excessive Gestational Weight Gain in Overweight and Obese Women [Inset Callout \#1 here]

The Institute of Medicine (2009) recommends an individualized gestational weight gain pattern for each pregnant woman based on her pre-pregnancy body mass index (BMI). For instance, the optimal weight gain during pregnancy for a normal weight woman (pre-pregnancy $\mathrm{BMI}=18.5-24.9$ ) is $25-35$ lbs. About $38 \%$ to $55 \%$ of pregnant women, however, gain more weight than what is recommended, and overweight and obese pregnant women are especially at risk for excessive gestational weight gain (EGWG) (Deputy, Sharma, \& Kim, 2015). EGWG increases a woman’s likelihood for hypertensive disorders, preeclampsia, impaired glucose tolerance, surgical manipulation during labor and delivery, and maternal weight retention during postpartum and years after birth (Fortner et al., 2009; Herring et al., 2009; Linné, Dye, Barkeling, \& Rössner, 2003; Rothberg et al., 2011; Stoland, Hopkins, \& Caughey, 2004). EGWG also increases the baby’s risks for being large for gestational age, preterm birth, fetal defects and abnormalities, and future overweight/obesity (Marchi, Berg, Dencker, Olander, \& Begley, 2015; Schack-Nielsen et al., 2010). The Right Weight Program, a self-monitoring enhanced lifestyle intervention involving eight weeks of self-monitoring of weight gain and lifestyle behaviors, was designed to help overweight and obese pregnant women gain the Institute of Medicine (IOM) recommended amount of gestational weight. The purpose of this study was to evaluate the feasibility and potential benefits of the program.

\section{Literature Review}

\section{Lifestyle Behaviors and EGWG}

Energy imbalance is the most common causal theory for EGWG. Increased total energy intake from diet and decreased energy expenditure from physical inactivity contribute to EGWG 
(Garner, Wardle, Poston, \& Croker, 2011). Foods with high fat, animal lipids, high energy density, and low dietary fiber are associated with EGWG (Maple-Brown, Romen, Thomas, Presley, \& Catalano, 2013; Olafsgottir, Skuladottier, Thorsdottir, Hauksson, \& Steingrimsdottier, 2006). Physical activity often declines as gestational age increases (Fell, Joseph, Armson, \&

Dodds, 2009). To date, practice guidelines for EGWG prevention are aimed at decreasing energy intake or improving energy imbalance. For instance, the American College of Obstetricians and Gynecologists (2005) and the American Dietetic Association (2008) recommend that care providers offer EGWG prevention education and counseling to pregnant women so that they will engage in healthy eating and physical activity. Interventions designed to enhance adherence to the IOM's recommended weight gain in pregnant women often are focused on changing lifestyle behaviors relative to nutrition, physical activity, or both (Garner, Wardle, Poston, \& Croker, 2011).

\section{Self-Monitoring of Lifestyle Behaviors}

Lifestyle behavior change is a complex process. Up to 40 different behavior change strategies for healthy eating and physical activity have been identified in previous studies (Michie et al., 2011). Among them, self-monitoring with at least one of the other four selfregulatory strategies (prompt intention, specific goal setting, review of behavioral goals, and feedback) are significantly more effective than interventions without these strategies in changing lifestyle behaviors (effect size .54 vs. .24 for healthy eating and .38 vs. .27 for physical activity) (Bird et al., 2013; Michie, Abraham, Whittington, \& McAteer, 2009). Among non-pregnant adults who perform self-monitoring, increased frequency of self-monitoring of diet, physical activity, or weight is linked to eating less fat and getting more exercise and to decreased waist circumference and weight loss (Burke et al., 2012; Conroy et al., 2011; Kong et al., 2012). Selfmonitoring may help lifestyle change because it increases self-awareness of specific behavior 
goals as well as empowers people to engage in self-evaluation and behavior modification (Donaldson, Fallows, \& Morris, 2013). Self-monitoring of one health behavior also has the potential to improve other health behaviors (Johnson \& Wardle, 2012).

\section{Self-Monitoring of Lifestyle Behaviors and EGWG}

To date, only a handful of published studies have incorporated self-monitoring in interventions to prevent EGWG. Two of those studies used self-monitoring of weight (Harrison, Teede, \& Lombard, 2014; Jeffries, Shub, Walker, Hiscock, \& Permezel, 2009); two selfmonitoring of diet (Guelinckx, Devlieger, Mullie, \& Vansant, 2010; Kinnunen et al., 2007); one self-monitoring of exercise (Hui et al., 2012); one self-monitoring of diet and physical activity (Mottola et al., 2010); and three included self-monitoring of weight, diet, and physical activity (Olson, Strawderman, \& Reed, 2004; Phelan et al., 2014; Rauh et al., 2013). These studies adopted self-monitoring as an intervention component or as a data collection method. Many of the writers stated that self-monitoring was encouraged or prompted. Information on how to conduct self-monitoring and self-monitoring adherence data was not reported. Previous selfmonitoring studies in non-pregnant overweight/obese adults reported compliance rates of $22 \%$ to 72\%, ranging from 6 weeks to 18 months of self-monitoring (Burke et al., 2012; Conroy et al., 2011; Donaldson et al., 2013; Hood et al., 2013; Kong et al., 2012; Steinberg et al., 2013; Wing et al., 2006). Information on how pregnant women respond to and comply with performing selfmonitoring has not been well documented in the literature.

\section{Study Purpose}

The purpose of this study was to evaluate the Right Weight Program, an eight-week selfmonitoring enhanced lifestyle intervention, designed to help overweight and obese pregnant women achieve IOM recommended weight gain. Specific aims were to evaluate feasibility (attrition, self-monitoring adherence, program safety, program satisfaction, participant feedback) 
and potential benefits (achievement of weight gain goal and improvement in nutrition and physical activity).

\section{Methods}

\section{Study Design}

The Right Weight Program was derived from the Health Action Process Approach Model (Schwarzer, 2008), in which action planning with goals and action control (self-monitoring, selfevaluation, and self-regulatory efforts) are theorized to change health behaviors. We adopted a progressive self-monitoring strategy in the Right Weight Program. Participants began with one self-monitoring behavior daily and gradually increased to three a day. This progressive strategy was to reduce subject burden in self-monitoring and to increase compliance. To evaluate the Right Weight Program, we used a one-group prospective design approved by the University’s Institutional Review Board.

\section{Participants}

Inclusion criteria were pregnant women who were 18 years or older, able to read and speak English, had a known pre-pregnancy BMI of 25-40, and were at 14-24 weeks of gestation. Subjects were excluded if they had multiple gestation, metabolic disorders (e.g., diabetes), obstetric complications (e.g., preterm labor, incompetent cervix), eating disorders, or psychiatric/mental health problems. Participants did not continue the study if they developed one of these exclusion conditions after enrollment.

Because our study was to assess feasibility in our population, we did not perform a priori sample size calculation. A previous study with 21 obese pregnant women and using food diaries and pedometers to enhance lifestyle change demonstrated positive outcomes in preventing EGWG (Schirazian, Monteith, Friedman, \& Rebarber, 2010). Our plan was to enroll 20-25 women to assess feasibility. 


\section{Setting}

Study participants were recruited from two prenatal clinics in the Midwest. These two clinics serve approximately 60-70 new pregnant patients a month, largely low-income women (60\% African Americans, 30\% Caucasians, and 10\% others). Study flyers were posted in the clinics. Recruitment occurred on days the clinics were open to new patients and after patients had completed an intake assessment by a clinic nurse. The clinic nurse referred overweight and obese women between 14 and 24 weeks gestation to the Right Weight Program; research staff then used a recruitment checklist to assess and verify eligibility and interest. A signed informed consent form was obtained from each participant.

The research staff, two doctoral nursing students and one master's-prepared dietician, received eight hours of training on the study procedure and protocol, motivational interview skills, communication with participants and research team members, and the REDCap ${ }^{\mathrm{TM}}$ data management system for data entry and subject tracking. They recruited study participants, conducted face-to-face sessions, collected weekly logs and intervention-related data on the same participants they had enrolled in order to ensure continuity and communication with participants. Program satisfaction surveys and semi-structured interviews were collected by the first author.

\section{Intervention}

Three major components were included in the Right Weight Program: weight gain and behavior performance goal achievement; self-monitoring of weight and behaviors; and brief patient education and performance feedback.

Weight gain and performance goals. Participants were instructed to achieve weekly weight gains of $\leq .5$ lbs. (for obese women; pre-pregnancy BMI 30 to 40 ) or $\leq .75$ lbs. (for overweight women; pre-pregnancy BMI 25 to 29.9) based on the IOM recommendations. There were four healthy eating and one walking performance goals: Eat 3 cups of vegetables a day; eat 
2 cups of fruits a day; eat low fat or fat free protein/dairy foods 3 times a day; have low sugar or sugar free drinks and beverages 3 times a day; and walk 30 minutes a day for at least 5 days a week and a total of 150 minutes a week.

Self-monitoring. In the first four weeks, study participants self-monitored weight (weeks 1 to 4), vegetable intake (week 2), fruit intake (week 3), and low fat or fat free protein/dairy intake (week 4) daily. Each participant was given a digital bathroom weight scale with a capacity of holding up to 400 lbs. Participants were taught to weigh themselves at the same time each day with light clothes on and no shoes worn and recorded to .1 pound. A food measurement cup was also given to each participant to help estimate food intake. In weeks 5 to 8, participants selfmonitored weight (weeks 5 to 8), walking (weeks 5 to 8), low fat or fat free protein/dairy food intake (week 7), and low sugar or sugar free drinks and beverages (week 8). A water bottle and a pedometer were given to each participant. Participants recorded the time, day, and selfmonitoring data daily on paper logs and submitted logs weekly via regular U.S. mail, email, or cell phone text to the research office. At the conclusion of the study, a participant received a \$20 grocery store gift card.

Brief patient education and performance feedback. Three individual face-to-face sessions were conducted in the clinics. The initial session (20 minutes) was given after study consent and before self-monitoring. During the initial session, a brief education about gestational weight gain and how to eat healthy and be physically active, supplemented with a written brochure, was given. Instructions on how to achieve goals, do daily self-monitoring and submit logs were also given. At the end of the 4th week, a booster session (20 minutes) was delivered. During the booster session, a summary report of the previous four weeks of self-monitoring data was reviewed with each participant, including whether or not weekly weight gain and behavior 
performance goals were met and suggestions for improvement. Participants were encouraged to use the BABY step strategy to promote behavior change. The BABY step stands for balance (have a balanced diet), action (take action to eat healthy and exercise now, not tomorrow), being aware (of physical and emotional changes), and yelling for help (have someone listen to or support you). The final session (30 to 40 minutes) occurred at the end of the 8th week. Another summary report of the previous four weeks of self-monitoring data was shared with each participant. A program satisfaction survey and a semi-structured interview were conducted. Data from semi-structured interviews are not reported in this paper.

\section{Data Collection and Measures}

Feasibility. We evaluated several dimensions of feasibility.

Attrition. We calculated attrition rates by the end of the $4^{\text {th }}$ week and the $8^{\text {th }}$ week.

Self-monitoring adherence. Two aspects of self-monitoring adherence were assessed: the percent of self-monitoring logs returned and the percent of weekly goals met.

Program safety. Participants who gained $\geq 6.5 \mathrm{lbs}$. or $\leq 1 \mathrm{lb}$. in four weeks were to be referred to their care providers for further assessment. Additionally, five potential physical and psychosocial side effects were assessed. In the self-monitoring logs, each participant rated at the end of each week: morning sickness and food craving (very strong, a little strong, not at all), stress (very high, a little high, not at all), household food shortage (enough, a little short, totally not enough), and mood (overall feel sad, a little sad, do not feel sad).

Program satisfaction. A program satisfaction survey, including nine questions (scored 1 to 10,10 being the highest satisfaction), assessed helpfulness of the program; program length; communication frequency; reminder calls or emails; feedback; overall satisfaction; and participant willingness to recommend the program to others. 
Participant Feedback. Two open-ended questions in each log asked participants to give written comments about what had helped them to achieve goals in a given week and what made it hard to achieve goals. Content analysis was conducted to identify major facilitators and barriers in the written comments of 120 weekly logs.

Potential benefits. The second aim of this study was to evaluate potential benefits of the Right Weight Program in achieving weight gain goals and in improving nutrition and physical activity. Data were collected at baseline (T1), the end of the $4^{\text {th }}$ week (T2) or the end of the $8^{\text {th }}$ week (T3).

Weight gain goal achieved. Weight was measured by using a digital weight scale (LifeSource MD ${ }^{\mathrm{TM}}$ ProFIT Precision Scale Model UC-321, A \& D medical, Milpitas, CA). Weight was measured twice and the average of the two measurements was used for analysis. We calculated first the amount of weight gain between T1 and T2 and T3 and T4. The amount of weight gain was then divided by the number of gestational weeks between two time points to produce average weekly weight gain. If the weekly weight gain was $\leq .75$ lbs. for overweight women and $\leq .5 \mathrm{lbs}$. for obese women, then the weight gain goal was achieved.

Nutrition. The Block Fat/Sugar/Fruit/Vegetable Screener (Lalonde et al., 2008) was used at T1 and T3 to assess dietary intake related to saturated fats, trans fats, added sugars, total sugars, fruits, and vegetables. This screener has 40 questions that assess frequency (how many days per week) and quantity of food intake and another 15 questions that ask participants to identify their usual habits in choosing regular or low fat, low carb, and low sugar foods.

Physical activity. The International Physical Activity Questionnaire-Short Version (Craig et al., 2003) was used at T1 and T3. This questionnaire contains seven items that assess how many days in the past seven days and how many minutes and hours that a person has done 
vigorous (e.g., aerobics or fast bicycling) and moderate (e.g., bicycling at a regular pace) physical activities, walking, and sitting. This instrument was used in a previous study on pregnant women (Bertolotto et al., 2010). We calculated total metabolic equivalents (METs) from all activities for each participant (vigorous $=8.0$ METs, moderate $=4.0$ METS, and walking = 3.3 METs). We then classified each participant's combined physical activities into low, moderate or high. Classifications were based on total METs and a required number of days or minutes for vigorous, moderate and walking activities based on the instrument's guidelines.

\section{Data Analysis}

Data were recorded in the REDCap ${ }^{\mathrm{TM}}$ database (Harris et al., 2009) and verified for accuracy by data management staff. Statistical analysis was performed by using SAS Statistical Software 9.4. Simple statistics (mean, standard deviation, frequency, and percentage) were conducted for the weekly log data and program satisfaction. We assessed the reasons why each dropout occurred. Those reasons were not related to outcomes (potential benefit variables) that we tested, therefore, we described the missingness as missing at random (MAR). The assumption of MAR usually depends on the reasons of missingness. Statistically, likelihood-based inference is valid using only observed data under MAR. Evaluation of potential benefits was based on preand post-intervention data for weight, nutrition, and physical activity. Generalized linear mixed model was performed for categorical variables and linear mixed model for continuous variables. If the assumptions for the linear mixed model were not satisfied, an appropriate transformation was conducted on variables. Logarithm transformation was performed for some nutrition variables and for METs, and their estimated means and standard errors were calculated. Based on each model, the odds ratio (OR) or Cohen's d was calculated as effect size.

\section{Results}




\section{Study Participants}

At baseline, 22 pregnant women were enrolled with a mean BMI of 30.7. Most women were Black, aged 20-29, had a high school or higher education, and were single, low-income, with young children at home. All of them had a cell phone, used text messaging and had a place to use the Internet. Of those who reported health practice behaviors before pregnancy $(n=16$, 72.7\%), many exercised $(n=11,68.8 \%)$ or had tried dieting $(n=7,43.8 \%)$. (Details in Table 1).

\section{Feasibility}

Attrition. Attrition rates were $27.3 \%$ and $40.9 \%$ at T2 and T3, respectively. Six women dropped out the study between $\mathrm{T} 1$ and $\mathrm{T} 2$, mostly due to no response or no time to continue the study. Three additional women withdrew from the study from T2 to T3 because they developed obstetric complications (preterm labor, twins, pregnancy-induced hypertension) and no longer meeting the inclusion criteria. These nine women were not different from those who completed the study in age, pre pregnancy BMI, ethnicity, or gestational age.

Self-monitoring adherence. All of the women who remained in the study returned the weekly logs. For weight gain, $50 \%(n=8 / 16)$ to $84.6 \%(n=11 / 13)$ of the participants met required goals from week 2 to week 8 . For nutrition, low or fat free goal was hardest to achieve ( $n=3 / 16,18.8 \%$ to $n=4 / 13,30.8 \%)$. Many women $(n=10 / 14,71.4 \%$ to $n=12 / 13,92.3 \%)$ met walking goals. (See Table 2 for details).

Program safety. Program safety data are included in Table 3. None of the women in this study gained $\geq 6.5 \mathrm{lbs}$. or $\leq 1 \mathrm{lb}$. in four weeks. The majority of participants did not report morning sickness, household food shortage, or sad mood across eight weeks. Some participants described their food cravings as very strong ( $n=2 / 13,15.4 \%$ to $n=6 / 13,46.2 \%$ ) or their stress levels as very high ( $n=2 / 18,11.1 \%$ to $4 / 13,30.8 \%$; higher in the last four weeks). 
Program satisfaction. Table 4 reports program satisfaction survey data by 14 participants (13 completed the study and one completed by T2). The overall mean satisfaction score was 7.7 (based on a 1 to 10 scale). We also identified areas indicating high satisfaction (scored at or above 8). Based on this method, "keep track of what I eat helps me eat more healthy foods” was highly endorsed (12/14 or $85.7 \%)$, and two other areas had the lowest endorsement of $64.3 \%(n=9 / 14)$ ("Keeping track of how many minutes I walk helps me be physically active” and "Eight weeks for the Right Weight Program is the right length, not too long and not too short”).

Participant feedback. Facilitators and barriers abstracted from written comments in the weekly logs are in Table 5. Strategies that helped participants achieve weekly goals included establishing a routine, setting up a reminder system, staying focused and watchful, having a positive attitude, and changing to better eating habits. Participants also identified barriers to goal achievement as being out of a routine (weekend, travel, going to parties), food cravings and change in taste, unavailability of food (not having enough fruits, vegetables, or low fat foods at home), physical and medical problems related to pregnancy (pelvic pain, heartburn, feet and knee hurt), eating out, work, and bad weather.

\section{Potential Benefits}

Potential benefit data are in Table 6. The odds of meeting the weight gain goal at T3 (4/13 or 31\%) were 1.91 times higher than the odds at T2 (3/16 or $19 \%)$ but the improvement was not statistically significant. There was a trend for participants to reduce intake of added sugars, saturated fats, and trans fats from T1 to T3, but only the reduction of trans fats almost reached statistical significance $(p=.06)$. The effect size for reducing trans fats $(.43)$ was larger than that for saturated fats (.24) or added sugars (.16). Intake of fruits or vegetables did not 
improve from T1 to T3. Physical activity significantly improved from T1 to T3 $(\mathrm{p}=.05)$ with increased percentages of moderate and high levels of physical activity $(n=13 / 21$ or $61.9 \%$ to $n=$ $13 / 13$ or $100 \%)$.

[Inset Callout \#2 here]

\section{Discussion}

We evaluated feasibility and potential benefits of the Right Weight Program, a selfmonitoring enhanced lifestyle intervention to help overweight and obese pregnant women gain the IOM recommended gestational weight. The intervention was viewed by participants as acceptable and helpful and did not cause untoward physical or psychological harms. Our intervention resulted in an improvement in physical activity (38\% increase in moderate and high levels) and a favorable trend in trans fat intake reduction (effect size of .43) and weight gain goal achievement. The intervention is worthy of further development and testing with a randomized controlled trial.

Further development of the Right Weight Program could focus on improving fruit/vegetable intake. The finding that fruit and vegetable intake did not increase from T1 to T3 was unexpected. This could be due to our small sample size as well as the fact that participants were required to self-monitor fruit and vegetable intake for only one week each. Some participants stated in their written comments that they did not have enough fruits and vegetables in their house. Food unavailability may also have contributed to the inability of the intervention to increase fruit/vegetable intake. From the self-reported log data, participants demonstrated better goal achievement in fruit and vegetable intake than low fat consumption. It might also be that participants had done well in fruit/vegetable consumption and there was little room for improvement. Nevertheless, to improve fruit/vegetable intake in the Right Weight Program we 
will need to increase sample size, lengthen self-monitoring for fruit/vegetable intake and provides participants information about how to deal with food unavailability issues.

Another improvement for the Right Weight Program is to reduce attrition. In our study, the attrition rates were $27.3 \%$ by T2 and $40.9 \%$ by T3. Previous studies, which ranged from 6 weeks to 18 months of self-monitoring, reported attrition rates of 22\% to $72 \%$ (Burke et al., 2012; Conroy et al., 2011; Donaldson et al., 2013; Hood et al., 2013; Kong et al., 2012;

Steinberg et al., 2013; Wing et al., 2006). The longer a study, the higher the attrition rate was. In our study, participants tended to drop out the study by T2 because they were lost to follow-up or had no time to participate in the study. The women who left the study between T2 and T3 were passively terminated because they developed obstetric complications and were no longer eligible. The \$20 compensation for completing our study might have been perceived by study participants as inadequate to compensate for their time and efforts. Greater compensation might improve attrition rates; however, participants in our study also received a weight scale, a food measurement cup, a water bottle and a pedometer as incentives. One positive note in our study was that participants who stayed in the study were committed to finishing weekly logs. To reduce attrition, future refinement should emphasize communication with participants about study expectations, stringent enrollment screening for potential OB and medical history, and support for participants throughout the intervention.

The Right Weight Program did not appear to cause major side effects in participants, and participants seemed to accept the program with a high satisfaction. Participants, however, reported persistent food cravings throughout the study. Previous studies found that $80 \%$ of pregnant women experienced food cravings and $45 \%$ developed new cravings for specific foods during pregnancy, with sweets being the most craved food (Belzer, Smulian, Lu, \& Tepper, 
2010; Farland, Rifas-Shiman, \& Gillman, 2015). Food cravings reported by the women in our study, therefore, may not reflect a program participation side-effect but a common phenomenon among pregnant women. Orloff and Horman (2014) stated that food cravings during pregnancy could be a potential modifiable determinant of energy intake and they are influenced by cultural and psychological factors, such as eating for two and ambivalence toward highly palatable and calorically dense food. Addressing food cravings and how to satisfy food cravings with healthy foods in the Right Weight Program may strengthen the intervention.

Higher percentages of the women in our study reported stress as very high in the last 4 weeks ( $n=2 / 13,15.4 \%$ to $n=4 / 13,30.8 \%)$ than in the first 4 weeks $(n=0 / 15,0 \%$ to $n=2 / 16$, 12.5\%). Increased stress could be from the advancement of pregnancy as reported by participants in their written comments, such as increased abdominal girth affected eating (e.g., heartburn) and walking (e.g., pain in joints and feet). However, increased stress could also be due to the demand of study participation. It will be important, in the future refinement of the intervention, to distinguish stress due to study participation versus other physical or psychosocial causes.

In our study, log data led to higher percentages of weekly weight gain goals achievement $(\mathrm{n}=5 / 18,27.8 \%$ to $\mathrm{n}=11 / 13,84.6 \%)$ than those calculated from measured weights by research staff at T2 $(n=3 / 16,18.8 \%)$ and T3 $(n=4 / 13,30.8 \%)$. One possible reason for the difference is that weekly self-reported weight gain reflected short-term weight changes in a week, and the staff-measured weights reflected changes over 4 weeks. However, social desirability might also have biased the log weight data. Although we gave participants instructions on how to weigh themselves at home (weigh self the same time each day, light clothes and no shoes), some weighed themselves in the afternoon or very early or very late in the morning. Others placed the weight scale in different locations in their homes. Future intervention improvement may consider 
using a weight scale that can store weight data and transmit them directly from home to research office if self-weighing data reported by participants will be used as a study outcome. Weighing instructions may also need to be reinforced.

Major limitations of this study were the single-group design and the small sample size; however, our study objective was to assess feasibility and potential benefits and results suggest the intervention is worthy of testing in a randomized controlled trial. The length of the study was only eight weeks, which may not have been long enough to produce sustained effects through the end of pregnancy. Further, this study included only overweight and obese pregnant women. Findings may not be applied to normal weight or underweight women.

In conclusion, the Right Weight Program produced more favorable outcomes, on the average, in helping overweight and obese pregnant women improve trans fat consumption and physical activity than achieve IOM recommended weigh gain. Improvement of the Right Weight Program should include increasing sample size, lengthening the intervention, adding more education and support for participants to reduce confounding effects from food cravings, stress and other physical, psychological and environmental barriers, as well as adding a comparison group. Further validation of the program will require a more stringent randomized controlled trial design.

[Inset Callout \#1 here]

\section{Implications for Practice}

The Right Weight Program supports theoretical and empirical underpinnings that selfmonitoring helps improve nutrition and physical activity and consequently help weight management. Nurses working in prenatal clinics should check each pregnant woman's weight during prenatal visits and discuss with the woman her weight gain patterns in relation to IOM 
recommendations. Waring et al. (2014) found that $26 \%$ of overweight and $45 \%$ of obese pregnant women did not recall receiving weight gain advice from their care providers. They also found that of those who did receive advice, 37\% of overweight and 39\% of obese pregnant women reported receiving weight gain advice from care providers that exceeded IOM recommendations. It is, therefore, important for nurses to make sure pregnant women receive verbal or written information and advice about the IOM recommended weight gain.

Nurses can use self-monitoring as a strategy to empower women to take charge of their weight management, healthy eating, and physical activity. For those pregnant women who are sedentary, self-monitoring of their walking may increase their levels of physical activity. Nurses also may use self-monitored data to verify client adherence to certain teaching or expected changes. Nurses can co-develop performance goals with pregnant women that are realistic for the women and provide feedback for them. Many clinics and healthcare systems use electronic health records (EHR). EHR allows patients to see their clinic visit records and communicate with their care providers through a password secured online portal. If the EHR is available in her practice location, a nurse can communication with pregnant women about self-monitoring data via EHR.

When teaching pregnant women about self-monitoring, nurses can adopt the strategies or facilitators offered by our study participants, such as setting up a routine, using a reminder system, staying focused and watchful, having a positive attitude, and changing to better eating habits. These strategies reflect self-regulatory efforts. Barriers reported by the women in our study may be used to develop anticipatory guidance for lifestyle change in general and selfmonitoring in specific. Prenatal teaching should include attention to women's reported barriers. For instance, encouraging pregnant women to satisfy their cravings with healthy food choices 
may reduce the likelihood of gaining unnecessary weight. A low frequency of eating out and more meal preparation at home are considered healthy eating behaviors (Shieh, Weaver, Newsome, Hanna, \& Mogos, 2015). Teaching pregnant women how to choose alternative foods when eating out is unavoidable may help pregnant women maintain healthy eating in various dining environments.

Nurses work alongside other care providers in clinics. They may collaborate with other providers and form a care team for pregnant women with complex needs for weight and lifestyle management. These women may benefit from an interprofessional practice team of nurses, physicians, psychologists, dieticians, and social workers. 


\section{References}

American College of Obstetricians and Gynecologists (2005). Committee Opinion. Obesity in pregnancy. Obstetrics \& Gynecology, 315, 671-675.

American Dietetic Association (2008). Position of the American Dietetic Association: Nutrition and lifestyle for a healthy pregnancy outcome. Journal of the American Dietetic Association, 108 (3), 553-561. doi: 10.1016/j.jada.2008.01.030

Bird, E.L., Baker, G., Mutrie, N., Ogilvie, D., Sahlqvist, S., \& Powell, J. (2013). Behavior change techniques used to promote walking and cycling: A systematic review. Health Psychology, 32 (8), 829-838. doi: 10.1037/a0032078

Belzer, L.M., Smulian, J.C., Lu, S.E, \& Tepper, B.J. (2010). Food cravings and intake of sweet foods in healthy pregnancy and mild gestational diabetes mellitus. A prospective study. Appetite, 55, 609-615. doi: 10.1016/j.appet.2010.09.014

Bertolotto, A., Volpe, L., Calianno, A., Pugliese, M.C., Lencioni, C., Resi, V.,...Di Cianni, G. (2010). Physical activity and dietary habits during pregnancy: effects on glucose tolerance. The Journal of Maternal-Fetal and Neonatal Medicine, 23(11), 1310-1314. dio: 10.3109/14767051003678150

Burke, L.E., Styn, M.A., Sereika, S.M., Conroy, M.B., Ye, L., Glanz, K....Ewing, L.J. (2012). Using mHealth technology to enhance self-monitoring for weight loss. A randomized trial. American Journal of Preventive Medicine, 43 (1), 20-26. doi.org/10.1061/j.amepre.2012.03.016

Conroy, M.B., Yang, K., Elci, O.U., Gabriel, K.P., Styn, M.A.,...Burke, L.E. (2011). Physical activity self-monitoring and weight loss: 6-month results of the SMART trial. Medicine \& Science in Sports \& Exercise, 43(8):1568-1574. doi: 10.1249/MSS.0b013e31820b9395

Craig, C.L., Marshall, A.L., Sjöström, M., Bauman, A.E., Booth, M.L., Ainsworth, B.E.... Oja, P. (2003). International Physical Activity Questionnaire: 12-Country Reliability and Validity. Medicine \& Science in Sports \& Exercise, 35 (8), 1381-1395.

Deputy, N.P., Sharma, A.J.., \& Kim, S.Y. (2015). Gestational weight gain, United States, 2012 and 2013. Morbidity and Mortality Weekly Report, 64 (43), 1215-1220.

Donaldson, E.L., Fallows, S., \& Morris, M. (2013). A text message based weight management intervention for overweight adults. Journal of Human Nutrition and Dietetics. doi: 10.1111/jhn.12096.

Farland, L.V., Rifas-Shiman, S.L., \& Gillman, M.W. (2015). Early pregnancy cravings, dietary intake, and development of abnormal glucose tolerance. Journal of Academy of Nutrition and Dietetics, 115, 1958-1964. http://dx.doi.org/10.1016/j/jand.2015.04.018

Fell, D.B., Joseph, K.S., Armson, B.A., \& Dodds, L. (2009). The impact of pregnancy on 
physical activity level. Maternal and Child Health Journal, 13 (50), 597-603. doi: 10.1007/s10995-008-0404-7

Fortner, T.R., Pekow, P., Solomon, C.G., Markenson, G., \& Chasan-Taber, L. (2009).

Pregnancy body mass index, gestational weight gain, and risk of hypertensive pregnancy among Latina women. American Journal of obstetrics \& Gynecology, 200, 167 e1-167 e7. doi:10.1016/j.ajog.2008.08.021

Garner, B., Wardle, J., Poston, L., \& Croker, H. (2011). Changing diet and physical activity to reduce gestational weight gain: A meta-analysis. Obesity Review, 12, e602-e620. doi: 10.1111/j.1467-789x.2011.00884.x

Guelinckx, I., Devlieger, R., Mullie, P., \& Vansant, G. (2010). Effects of lifestyle intervention on dietary habits, physical activity, and gestational weight gain in obese pregnant women: a randomized controlled trial. American Journal of Clinical Nutrition, 91, 373-380. doi: 10.3945/ajcn.2009.28166

Harris, P.A., Taylor, R., Thielke, R., Rayne, J., Gonzalez, N., Conde, J.G. (2009). Research electronic data capture (REDCap) - A metadata-driven methodology and workflow process for providing translational research informatics support. Journal of Biomedical Informatics, 42, 377-381.

Harrison, C.L., Lombard, C.B., \& Teede, H.L. (2014). Limiting postpartum weight retention through early antenatal intervention: the HeLP-her randomized controlled trial. International Journal of Behavioral Nutrition and Physical Activity: 11:134. doi: 10.1186/s12966-014-0134-8

Herring, S.J., Oken, E., Rifas-Shiman, S.L., Rich-Edwards, J.W., Stuebe, A.M., Kleinman, K....Gillman, M.W. (2009). Weight gain in pregnancy and risk of maternal hyperglycemia. American Journal of Obstetrics and Gynecology, 201(1), 61.e1-61.e7. doi: 10.1016/j.ajog.2009.01.039

Hood, M.M., Corsica, J., Cvebgris, J., \& Wyatt, J. (2013). Impact of a brief self-monitoring intervention on weight change and CPAP adherence in patients with obstructive sleep apnea. Journal of Psychosomatic Research, 74, 170-174. doi:

10.1016/j.jpsychores.2012.12.006

Hui, A.L., Back, L., Ludwig, S., Gardiner, P., Sevenhuysen, G., Dean, H., Sellers, E....Shen, G.X. (2012). Lifestyle intervention on diet and exercise reduced excessive gestational weight gain in pregnant women under a randomized controlled trial. BJOG: An International Journal of Obstetrics \& Gynecology, 119 (1), 70-77. doi: 10.1111/j.14710528.2011.03184.x

Institute of Medicine (2009). Weight gain during pregnancy: Reexamining the guidelines. Washington, DC: The National Academies Press. 
Jeffries, K., Shub, A., Walker, S.P., Hiscock, R., \& Permezel, M. (2009). Reducing excessive weight gain in pregnancy: a randomized controlled trial. Medical Journal of Australia, 191, 429-433.

Johnson, E., \& Wardle, P.J. (2012). Dietary restraint and self-regulation in eating behavior. International Journal of Obesity, 36, 665-674. doi:10.1038/ijo.2011.156

Kinnunen, T.I., Pasanen, M., Aittasalo, M., Hilakivi-Clark, L., Weiderpass, E., \& Luoto, R. (2007). Preventing excessive weight gain during pregnancy-a controlled trial in primary health care. European Journal of Clinical Nutrition, 61, 884-891. doi: 10.1038/sj.ejcn.1602602

Kong, A., Beresford, S.A.A., Alfano, K.M., Forster-Schubert, K.E., Neuhouser, M.L., Johnson, D.B....McTiernan, A. (2012). Self-monitoring and eating-related behaviors are associated with 12-month weight loss in postmenopausal overweight-to-obese women. Journal of the Academy of Nutrition and Dietetics, 112, 1428-1435. doi: 10.1016/j.jand.2012.05.014

Lalonde, I., Graham, M., Slovinec-D'Angelo, M., Beaton, L., Brown, J., \& Block, T. (2008). Validation of the Block Fat/Sugar/Fruit/Vegetable Screener in a Cardiac Rehabilitation Setting. Journal of Cardiopulmonary Rehabilitation \& Prevention, 28 (5), 340. doi: 10.1097/01.HCR.0000336162.26720.95

Linné, Y; Dye, L., Barkeling, B, \& Rössner, S. (2003). Weight development over time in parous women-The SPAWN Study-15 ys follow-up. International Journal of Obesity, 27,1516-1522. doi:10.1038/sj.ijo.0802441

Maple-Brown, L.J., Romen, N.M., Thomas, A., Presley, L.H., \& Catalano, P.M. (2013). Perinatal factors relating to changes in maternal body fat in later gestational. Journal of Perinatology, 33, 934-938. doi: 10.1038/jp.2013.109

Marchi, J., Berg, M., Dencker, A., Olander, E.K., \& Begley, C. (2015). Risks associated with obesity in pregnancy, for the mother and baby: A systemic review. Obesity Review, 16, 621-638. doi: 10.1111/obr.12288

Michie, S., Ashford, S., Sniehotta, F., Dombrowski, S.U., Bishop, A., \& French, D. P. (2011). A refined taxonomy of behavior change techniques to help people change their physical activity and healthy eating behaviors: The CALO-RE taxonomy. Psychology and Health, 26 (11), 1479-1498. doi: 10.1080/08870446.2010.540664

Michie, S., Abraham, C., Whittington, C., \& McAteer, J. (2009). Effective techniques in healthy eating and physical activity interventions: A meta-regression. Health Psychology, 28 (6), 690-701. doi: 10.1037/a0016136

Mottola, M.F., Giroux, I., Gratton, R., Hammond, J. Hanley, A., Harris, S....Sopper, M.M. 
(2010). Nutrition and exercise prevent excess weight gain in overweight pregnant women. Medicine \& Science in Sports \& Exercise, 42(2), 265-272. doi:

10.1249/MSS.0b013e3181b5419a

Olafsgottir, A.S., Skuladottier, G.V., Thorsdottir, I., Hauksson, A., \& Steingrimsdottier, L. (2006). Maternal diet in early and late pregnancy in relation to weight gain. International Journal of Obesity, 30, 492-499. doi: 10.1038/sj.ijo.0803184

Olson, C.M., Strawderman, M.S., \& Reed, R. G. (2004). Efficacy of an intervention to prevent excessive gestational weight gain. American Journal of Obstetrics and Gynecology, 191, 530-536. doi: 10.1016/j.ajog.2004.01.027

Orloff, N.C., \& hormes, J.M. (2014). Pickles and ice cream! Food cravings in pregnancy: hypotheses, preliminary evidence, and derections for future research. Frontier in Psychology, 5, Article 1076, 1-15. doi: 10.3389/fpsyg.2014.01076

Phelan, S., Phipps, M.G., Abrams, B., Darroch, F., Grantham, K., Schaffner, A., \& Wing, R. (2014). Does behavioral intervention in pregnancy reduce postpartum weight retention? Twelve-month outcomes of the Fit for Delivery randomized trial. American Journal of Clinical Nutrition, 99, 302-311.

Rauh, K., Gabriel, E., Kerschbaum, E., Schuster, T., von Kries, R., Amann-Gassner, U, \& Hauner, H. (2014). Safety and efficacy of a lifestyle intervention for pregnant women to prevent excessive maternal weight gain: A cluster-randomized controlled trial. BMC Pregnancy and Childbirth, 13: 151. doi: 10.1186/1471-2393-13-151

Rothberg, B.E., Magriples, U., Kershaw, T.S., Rising, S.S. \& Ickovics, J.R. (2011). Gestational weight gain and subsequent postpartum weight loss among young, low-income ethnic minority women. American Journal of Obstetrics \& Gynecology, 204, 52.e1-52.e11. doi: 10.1016/j.ajog.2010.08.028

Schack-Nielsen, L., Michaelsen, K.F., Gamborg, M., Mortensen, E.L., \& Sorensen, T.I.A. (2010). Gestational weight gain in relation to offspring body mass and obesity from infancy through adulthood. International Journal of Obesity, 31(4), 67-74. doi: 10.1038/ijo.2009.206

Schirazian, T., Monteith, S., Friedman, F., \& Rebarber, A. (2010). Lifestyle modification program decreases pregnancy weight gain in obese women. American Journal of Perinatology, 27(5), 411-414. doi: http://dx.doi.org/10.1055/s-0029-1243368

Schwarzer, R. (2008). Modeling health behavior change: How to predict and modify the adoption and maintenance of health behaviors. Applied Psychology: An International Review, 57, 1-29.

Shieh, C., Weaver, M.T., Hanna, K., Newsome, K., \& Mogos, M. (2015). Association of self- 
efficacy and self-regulation with nutrition and exercise behaviors in a community sample of adults. Journal of Community Health Nursing, 32 (4), 199-211. doi:

10.1080/07370016.2015.1087262

Steinberg, D.M., Levine, E.L., Askew, S., Foley, P., \& Bennett, G.G. (2013). Daily text messaging for weight control among racial and ethnic minority women: Randomized controlled pilot study. Journal of Medical Internet Research, 15 (11), e244.

doi:10.2196/jmir.2844

Stotland, N.E., Hopkins, L.M., Caughey, A.B. (2004). Gestational weight gain, macrosomia, and risk of cesarean birth in nondiabetic nulliparas. Obstetrics \& Gynecology, 104, 671-677. doi: 10.1097/01.AOG.0000139515.97799.f6

Waring, M.E., Moore-Simas, T.A., Barnes, K.C., Terk, D., Baran, I., Pagoto, S.L., \& Rosa, M.C. (2014). Patient report of guideline-congruent gestational weight gain advice from prenatal care providers: differences by prepregnancy BMI. Birth. 41(4), 353-359.

Wing, R.R., Tate, D.F., Gorin, A.A., Raynor, H.A., \& Fava, L. (2006). A self-regulation program for maintenance of weight loss. The New England Journal of Medicine, 355 (15), 15631571. doi: 10.1056/NEJMoa061883 
Table 1. Demographic, Obstetric, and Health History of Study Participants $(N=22)$

\begin{tabular}{|c|c|c|c|}
\hline Variables & $\mathrm{N}(\%)$ & Variables & $\mathrm{N}(\%)$ \\
\hline Race & & Number of Pregnancy including current one & \\
\hline Black & 14 (63.6) & $0-1$ & $8(36.4)$ \\
\hline White & $4(18.2)$ & $2-3$ & $7(31.8)$ \\
\hline Other Races & $4(18.2)$ & $\geq 3$ & $7(31.8)$ \\
\hline Age (years) & & Pre-pregnancy BMI, Mean (SD) & $30.7(3.0)$ \\
\hline$<20$ & $2(9.1)$ & Pre-pregnancy BMI & \\
\hline $20-29$ & $11(50.0)$ & Obese $(\geq 30)$ & $12(54.5)$ \\
\hline $30-39$ & $8(36.4)$ & Overweight (25 -29.9) & $10(45.5)$ \\
\hline$\geq 40$ & $1(4.5)$ & & \\
\hline Education & & Gestational Age (wks), Mean (SD) & $17.9(3.1)$ \\
\hline Grade $<=12$ & $10(45.5)$ & Gestational Age (wks) & \\
\hline Some College/Associate/Bachelor & $9(40.9)$ & $14-19.9$ & $17(77.3)$ \\
\hline Graduate degree & $3(13.6)$ & $20-24$ & $5(22.7)$ \\
\hline Marital Status & & Youngest Child When Pregnant, N = 14 & \\
\hline Married & $8(36.4)$ & $\leq 12$ months old & $5(38.5)$ \\
\hline Single & $13(59.1)$ & $\overline{1} 3-36$ months old & $3(23.1)$ \\
\hline Divorced/Separated & $1(4.5)$ & $\geq 37$ months & 5 (38.5) \\
\hline Household Income & & Health Practice Before Pregnancy, N = 16 & \\
\hline Less than $\$ 25 \mathrm{k}$ a year & $9(40.9)$ & Dieting & $7(43.8)$ \\
\hline$\$ 25 k$ - \$50k a year & $9(40.9)$ & Exercising & $11(68.8)$ \\
\hline$\$ 50 \mathrm{k}$ - \$75k a year & $3(13.6)$ & Taking Prescription Meds & $2(12.5)$ \\
\hline \multirow[t]{2}{*}{ More than $\$ 100 \mathrm{k}$ a year } & $1(4.5)$ & Blood Pressure Visit & $1(6.3)$ \\
\hline & & Depression Visit & $1(6.3)$ \\
\hline Work Outside Home & & Dental Visit & $8(50.0)$ \\
\hline Yes & $11(50.0)$ & Smoke cigarettes & $6(37.5)$ \\
\hline No & $11(50.0)$ & & \\
\hline
\end{tabular}


Table 2. Attrition and Adherence to Self-Monitoring and Weekly Goals based on Weekly Log Data (Baseline N = 22)

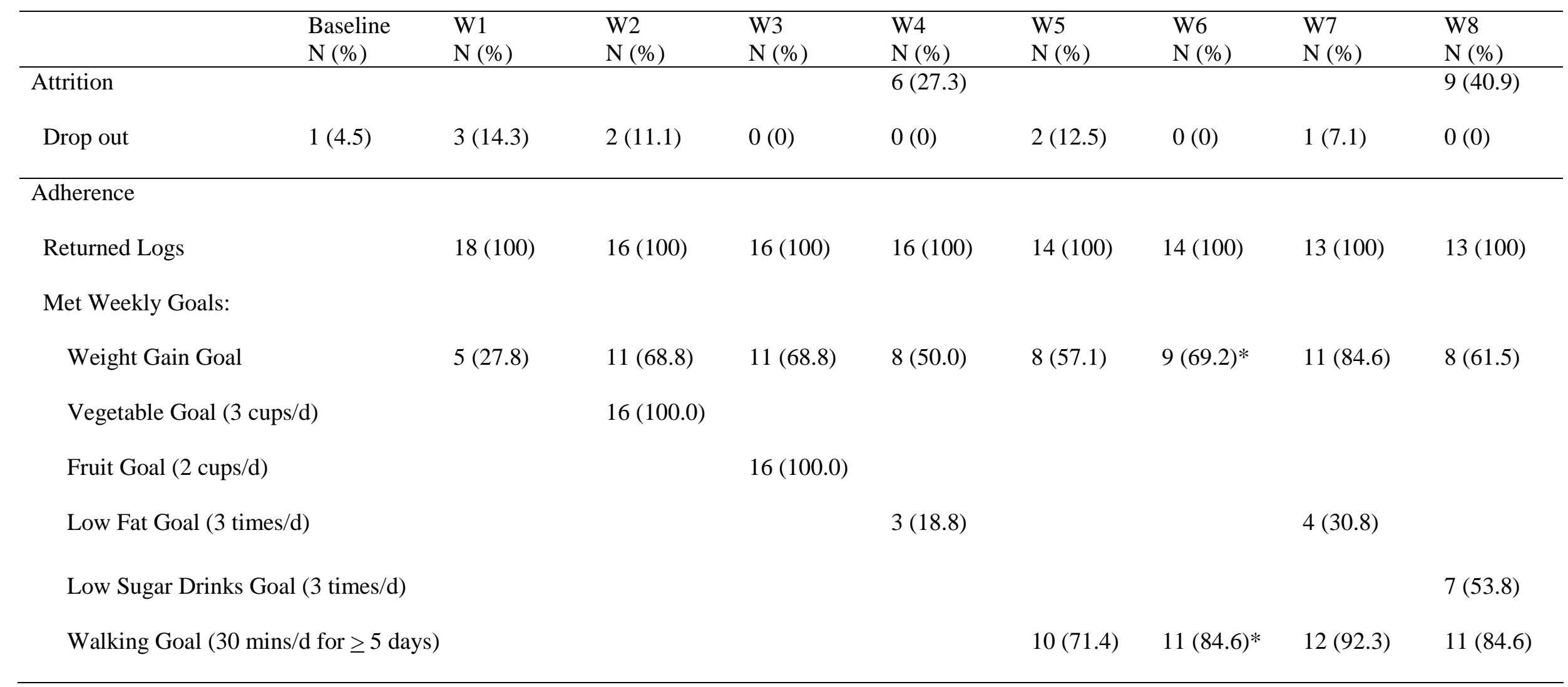

*one participant had missing data 
Table 3. Self-Report Weight Change and Potential Side Effects during Self-Monitoring Based on Log Data

\begin{tabular}{|c|c|c|c|c|c|c|c|c|}
\hline Variables & $\begin{array}{l}\text { W1 } \\
\text { M (SD) }\end{array}$ & $\begin{array}{l}\text { W2 } \\
M(S D)\end{array}$ & $\begin{array}{l}\text { W3 } \\
\text { M (SD) }\end{array}$ & $\begin{array}{l}\text { W4 } \\
\text { M (SD) }\end{array}$ & $\begin{array}{l}\text { W5 } \\
\text { M (SD) }\end{array}$ & $\begin{array}{l}\text { W6 } \\
\text { M (SD) }\end{array}$ & $\begin{array}{l}\text { W7 } \\
M(S D)\end{array}$ & $\begin{array}{l}\text { W8 } \\
\text { M (SD) }\end{array}$ \\
\hline \multicolumn{9}{|l|}{ Weight Fluctuation, lbs } \\
\hline Overweight & $2.2(2.5)$ & $0.6(1.7)$ & $1.1(2.1)$ & $-0.2(0.8)$ & $1.9(2.1)$ & $-0.4(2.6)$ & $0.1(0.8)$ & $0.6(1.1)$ \\
\hline Obese & $1.9(2.1)$ & $-0.1(2.2)$ & $0.2(1.2)$ & $1.5(1.3)$ & $0.5(0.8)$ & $0.0(1.6)$ & $0.3(0.6)$ & $1.2(1.4)$ \\
\hline \multicolumn{9}{|l|}{ Weekly Weight Gain, lbs } \\
\hline Overweight & $1.4(1.2)$ & $0.1(0.8)$ & $0.2(1.1)$ & $-0.6(0.5)$ & $0.8(1.8)$ & $-1.1(2.5)$ & $0.0(0.6)$ & $0.1(0.5)$ \\
\hline \multirow[t]{2}{*}{ Obese } & $1.4(1.9)$ & $0.0(1.5)$ & $-0.2(1.1)$ & $1.1(1.1)$ & $0.3(0.8)$ & $0.1(1.5)$ & $0.2(0.8)$ & $0.7(0.8)$ \\
\hline & $\mathrm{N}(\%)$ & $\mathrm{N}(\%)^{*}$ & $\mathrm{~N}(\%)^{*}$ & $\mathrm{~N}(\%)$ & $\mathrm{N}(\%)^{*}$ & $\mathrm{~N}(\%)^{*}$ & N (\%) & $\mathrm{N}(\%)$ \\
\hline \multicolumn{9}{|l|}{ Morning Sickness } \\
\hline Very Strong & $0(0)$ & $0(0)$ & $0(0)$ & $1(6.3)$ & $0(0)$ & $1(7.7)$ & $0(0)$ & $1(7.7)$ \\
\hline A little bit strong & $6(33.3)$ & $4(26.7)$ & $2(12.5)$ & $4(25.0)$ & $3(23.1)$ & $2(15.4)$ & $3(23.1)$ & $1(7.7)$ \\
\hline Not at all & $12(66.7)$ & $11(73.3)$ & $14(87.5)$ & $11(68.8)$ & $10(76.9)$ & $10(76.9)$ & $10(76.9)$ & $11(84.6)$ \\
\hline \multicolumn{9}{|l|}{ Craving } \\
\hline Very Strong & $5(27.8)$ & $5(33.3)$ & $5(33.3)$ & $3(18.8)$ & $6(46.2)$ & $2(15.4)$ & $4(30.8)$ & $2(15.4)$ \\
\hline A little bit strong & $8(44.4)$ & $5(33.3)$ & $5(33.3)$ & $8(50.0)$ & $2(15.4)$ & $5(38.5)$ & $6(46.2)$ & $5(38.5)$ \\
\hline Not at all & $5(27.8)$ & $5(33.3)$ & $5(33.3)$ & $5(31.3)$ & $5(38.5)$ & $6(46.2)$ & $3(23.1)$ & $6(46.2)$ \\
\hline \multicolumn{9}{|l|}{ Stress } \\
\hline Very High & $2(11.1)$ & $0(0)$ & $2(12.5)$ & $2(12.5)$ & $3(21.4)$ & $4(30.8)$ & $2(15.4)$ & $3(23.1)$ \\
\hline A little bit high & $4(22.2)$ & 7 (46.7) & $3(18.8)$ & $8(50.0)$ & $3(21.4)$ & 4 (30.8) & $7(53.8)$ & $4(30.8)$ \\
\hline Not at all & $12(66.7)$ & $8(53.3)$ & $11(68.8)$ & $6(37.5)$ & $8(57.1)$ & $5(38.5)$ & $4(30.8)$ & $6(46.2)$ \\
\hline \multicolumn{9}{|l|}{ Household food shortage } \\
\hline Enough food & 15 (83.3) & $6(40.0)$ & $12(75.0)$ & $13(81.3)$ & 13 (92.9) & $11(84.6)$ & $11(84.6)$ & $11(84.6)$ \\
\hline A little bit short & $3(16.7)$ & $7(46.7)$ & $4(25.0)$ & $2(12.5)$ & $1(7.1)$ & $2(15.4)$ & $0(0)$ & $2(15.4)$ \\
\hline Totally not enough & $0(0)$ & $2(13.3)$ & $0(0)$ & $1(6.3)$ & $0(0)$ & $0(0)$ & $2(15.4)$ & $0(0)$ \\
\hline \multicolumn{9}{|l|}{ Sad Mood } \\
\hline Overall feel sad & $0(0)$ & $0(0)$ & $1(6.3)$ & $2(12.5)$ & $1(7.1)$ & $2(15.4)$ & $2(15.4)$ & $0(0)$ \\
\hline A little bit sad & $3(16.7)$ & $5(33.3)$ & $3(18.8)$ & $4(25.0)$ & $3(21.4)$ & $2(15.4)$ & $3(23.1)$ & $4(30.8)$ \\
\hline Do not feel sad & $15(83.3)$ & $10(66.7)$ & $12(75.0)$ & $1062.5)$ & $10(71.4)$ & $9(69.2)$ & $8(61.5)$ & $9(69.2)$ \\
\hline
\end{tabular}

*Indicates one participant missed one or more items in the week 
Table 4. Program Satisfaction by Study Participants: How Agreeable with Survey Questions, Possible Scores from 1 to $10(N=14)$

\begin{tabular}{|c|c|c|c|}
\hline \multirow[t]{2}{*}{ Survey Items } & \multicolumn{2}{|c|}{ Scores } & \multirow{2}{*}{$\begin{array}{l}\text { High Satisfaction } \\
\text { (Scores } \geq 8) \\
n(\%)\end{array}$} \\
\hline & Range & M (SD) & \\
\hline $\begin{array}{l}\text { Right Weight Program helps me gain the right } \\
\text { amount of pregnancy weight }\end{array}$ & $4-10$ & $7.9(2.1)$ & $10(71.4)$ \\
\hline $\begin{array}{l}\text { Keeping track of what I eat helps me eat more } \\
\text { healthy foods }\end{array}$ & $6-10$ & $8.9(1.2)$ & 12 (85.7) \\
\hline $\begin{array}{l}\text { Keeping track of how many minutes I walk } \\
\text { helps me be physically active }\end{array}$ & $3-10$ & $8.1(2.1)$ & 9 (64.3) \\
\hline $\begin{array}{l}\text { Eight weeks for the Right Weight Program is } \\
\text { the right length, not too long and not too short }\end{array}$ & $5-10$ & $8.2(1.8)$ & $9(64.3)$ \\
\hline $\begin{array}{l}\text { Too many phone calls, emails, or text messages } \\
\text { sent by the Right Weight program }\end{array}$ & $1-10$ & $7.2(2.8)^{*}$ & $10(71.4)^{*}$ \\
\hline $\begin{array}{l}\text { I like to have a reminder phone call, email or } \\
\text { text message to keep me on track }\end{array}$ & $1-10$ & $7.6(3.2)$ & $10(71.4)$ \\
\hline $\begin{array}{l}\text { The feedback I got at the end of the } 4^{\text {th }} \text { and } 8^{\text {th }} \\
\text { weeks is helpful to me }\end{array}$ & $5-10$ & $8.4(1.9)$ & $10(71.4)$ \\
\hline $\begin{array}{l}\text { I am satisfied with what the Right Weight } \\
\text { Program has offered me }\end{array}$ & $5-10$ & 8.5 (1.9) & $10(71.4)$ \\
\hline $\begin{array}{l}\text { I will recommend Right Weight Program to } \\
\text { other pregnant women }\end{array}$ & $5-10$ & $8.6(1.9)$ & $10(71.4)$ \\
\hline Group Mean & 4.2-9.3 & 7.7 (1.5) & $10(71.4)$ \\
\hline
\end{tabular}

*this item was reversely calculated; One participant filled out the survey at the end of the $4^{\text {th }}$ week 
Table 5. Summative Themes: Facilitators and Barriers to Achieving Weekly Goals

Things that help to reach goals (Facilitators) Things that make it hard to reach goals (Barriers)

\section{Establishing a Routine}

- Start working for 30 minutes every morning

- Using my routine, weighing myself in the morning

- Walking getting up early

- Having a routine helped a lot

Setting up a Reminder System

- $\quad$ Setting an alarm every morning

- Write down in calendar

- Keeping scale next to my bed

- Keeping the pedometer on me helped

- Keeping the paper beside bed

Staying Focus and Watchful

- Focused on what I ate

- Watched what I ate

- Watch how much I was eating and make sure I had fruit for a snack

\section{Having a Positive Attitude}

- Do my best to follow the work sheet

- Keep thinking to myself I need to eat healthy because I have a child now

- Motived to stay active

- Determination

\section{Changing to Better Eating Habits}

- Making salads, sandwiches and stir fry helped with my goals

- Eating a fruit with each meal

- Eat lots of yogurt often, so that helped to reach my goal
Out of a Routine

- It was hard over the weekend. I forgot about it and sleep in

- Out of town

- Weekend plan away from home

- Eating late time

- Multiple retirement parties with bad food choices

- I had a very busy schedule this week

Craving and Change in Taste

- I craved every restaurant in town

- Hungry all the time

- Stressful day, gave into craving

- It was hard because making sure I added fruit even when I had no taste for it

Unavailability of Food

- Not having enough fruit to eat made it hard

- No low fat dairy protein foods

- Did not have money to buy food

- Sometimes the vegetable that I want to eat was not available

- Not having enough veggie in the house

Physical and Medical Problems

- Pelvic pain

- Sick for two days

- The further I get in my pregnancy, it is harder to move.

- Preterm labor

- Sick, nausea and heartburn

- Feet and knee hurt

- So tired to walk 
- Lots of fruits in the house

- Precut vege

- Planned meal

- Diversifying meals

- Eat the right food

- Sticking to the diet plan

- Eat lots of good food and go to the gym

- Making fruit and yogurt everyday
Work

- Working extra hours of second job didn't allow me to get workouts

- Finding time to eat

Eating out

- Ate out a lot

- Eating too much fast food

Bad weather

- It's hot out makes me miserable 
Table 6. Potential Program Benefits Related to Weight, Nutrition, and Physical Activity

\begin{tabular}{|c|c|c|c|c|c|c|c|c|}
\hline \multirow[t]{2}{*}{ Variables } & \multicolumn{2}{|c|}{$\begin{array}{c}\text { Baseline } \\
\mathrm{N}=21\end{array}$} & \multicolumn{2}{|c|}{$\begin{array}{c}\text { Booster (4 weeks) } \\
\quad \mathrm{N}=16\end{array}$} & \multicolumn{2}{|c|}{$\begin{array}{l}\text { End of Study (8 weeks) } \\
\mathrm{N}=13\end{array}$} & \multirow[b]{2}{*}{ P-value } & \multirow{2}{*}{$\begin{array}{l}\text { Effect Size } \\
(95 \% \mathrm{CI})\end{array}$} \\
\hline & $\mathrm{M}(\mathrm{SD})$ & Est M (SE) ${ }^{\mathrm{b}}$ & $\mathrm{M}(\mathrm{SD})$ & Est M (SE) ${ }^{\mathrm{b}}$ & M (SD) & Est M (SE) & & \\
\hline Weight Gain Goal Met, N (\%) & & & $3(18.8 \%)$ & & $4(30.8 \%)$ & & 0.46 & $1.91(0.35,10.49)$ \\
\hline \multicolumn{9}{|l|}{ Nutrition } \\
\hline Added Sugars grams ${ }^{\mathrm{a}}$ & $67.7(87.8)$ & $3.5(0.3)$ & & & $41.9(50.8)$ & $3.4(0.3)$ & 0.28 & $0.24(-0.46,0.94)$ \\
\hline Total Sugars grams ${ }^{a}$ & $109.5(90.9)$ & $4.4(0.2)$ & & & $89.6(58.1)$ & $4.5(0.2)$ & 0.85 & $-0.04(-0.74,0.66)$ \\
\hline Saturated Fat grams ${ }^{\mathrm{a}}$ & $27.9(21.9)$ & $3.1(0.2)$ & & & $22.7(12.0)$ & $3.0(0.2)$ & 0.47 & $0.16(-0.54,0.86)$ \\
\hline Trans Fat grams $^{\mathrm{a}}$ & $5.9(8.2)$ & $1.2(0.3)$ & & & $3.4(3.2)$ & $0.7(0.3)$ & 0.06 & $0.43(-0.28,1.13)$ \\
\hline Fruit/Fruit Juice Intake cup & $1.7(1.0)$ & $1.7(0.2)$ & & & $1.7(.9)$ & $1.7(0.2)$ & 0.97 & $-0.007(-0.71,0.69)$ \\
\hline Total Fruit/Vege cup & $3.5(2.1)$ & $3.5(0.5)$ & & & $3.2(1.3)$ & $3.2(0.3)$ & 0.39 & $-0.19(-0.89,0.51)$ \\
\hline Vegetable Intake cup & $1.9(1.4)$ & $1.9(0.3)$ & & & $1.5(.6)$ & $1.5(0.2)$ & 0.20 & $-0.28(-0.98,0.42)$ \\
\hline \multicolumn{9}{|l|}{ Physical Activity, N (\%) } \\
\hline Low & $8(38.1 \%)$ & & & & $0(0 \%)$ & & $0.05^{*}$ & \\
\hline Moderate & $11(52.4 \%)$ & & & & $10(76.9 \%)$ & & & \\
\hline High & $2(9.5 \%)$ & & & & $3(23.1 \%)$ & & & \\
\hline Physical Activity (MET) ${ }^{\mathrm{a}}$ & $\begin{array}{l}4560.4 \\
(7929.2)\end{array}$ & $7.4(0.3)$ & & & $\begin{array}{l}4412.0 \\
(4591.8)\end{array}$ & $8.0(0.3)$ & 0.09 & \\
\hline
\end{tabular}

Note: Generalized Linear Mixed Model was used for categorical variables and odds ratio was provided for effect size. Linear Mixed Model was performed

for continuous variables and Cohen's d was provided for effect size. Positive Cohen’s d reflects the expected result. Average gestational weeks were $17.8 \pm 3.1$ (baseline), $24.8 \pm 4.6$ (booster), and $30.9 \pm 4.7$ (end of study)

a: Logarithm transformation was performed

b: Est M: estimated mean; SE: standard error; values calculated from logarithm transformation

MET: Metabolic equivalents

${ }^{*}$ p-value $<.05$ 